\title{
Haploid Induction in Triticale $\times$ Wheat and Wheat $\times$ Rye Derivatives Following Imperata cylindrica-Mediated Chromosome Elimination Approach
}

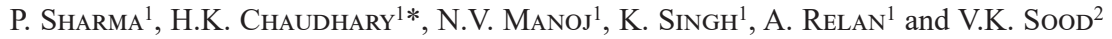 \\ ${ }^{1}$ The Molecular Cytogenetics and Tissue Culture Laboratory, Department of Crop Improvement, \\ CSK HP Agricultural University, Palampur (H.P), India-176062 \\ ${ }^{2}$ Department of Crop Improvement, CSK HP Agricultural University, Palampur (H.P), India-176062
}

(Received 27 May 2019; Accepted 9 September 2019;

Communicated by S.P. Baenziger)

The present research endeavor was undertaken to depict the response of different generations viz., $\mathrm{F}_{1}, \mathrm{~F}_{2}, \mathrm{BC}_{1} \mathrm{~F}_{1}, \mathrm{BC}_{1} \mathrm{~F}_{2}, \mathrm{BC}_{1} \mathrm{~F}_{3}, \mathrm{BC}_{1} \mathrm{~F}_{4}$ and $\mathrm{BC}_{1} \mathrm{~F}_{5}$ of triticale $\times$ wheat and wheat $\times$ rye hybrids towards the different parameters of haploid induction. The experimental material included the different generations obtained utilizing five genotypes of triticale (DT-123, DT-126, TL-2900, TL-2908 and TL-9335), four genotypes of Himalayan rye (Karoki rye, shanoor rye, tino rye and triloki rye) with various elite bread wheat genotypes as parents in wide hybridization programme. The triticale $\times$ wheat and wheat $\times$ rye recombinants were further subjected to Imperata cylindrica-mediated chromosome elimination approach of doubled haploidy breeding. The variability in the haploid induction parameters was observed to be under genetic control for embryo formation and regeneration, while pseudoseed formation was only affected by auxin treatment. Among the different generations, the backcross generations viz., $\mathrm{BC}_{1} \mathrm{~F}_{1}$ and $\mathrm{BC}_{1} \mathrm{~F}_{2}$ were found to exhibit significant positive response towards haploid induction parameters in both triticale $\times$ wheat and wheat $\times$ rye hybridization. Knowledge of effective generation for haploid induction in triticale $\times$ wheat and wheat $\times$ rye hybridization not only saved the time and energy but also enhanced the efficiency of haploid induction.

Keywords: Triticale $\times$ wheat, wheat $\times$ rye, Imperata cylindrica, haploid induction

\section{Introduction}

Wheat (Triticum aestivum L. em Thell) that belongs to poaceae family, is one of the most important food crops consumed throughout the world. Keeping this in consideration and due to increased prosperity, the productivity of wheat must be increased. Moreover, the frequent outbreaks of new races of pathogens and other stresses frequently demand the new improved cultivars at a faster rate than the past. Hence, there is a dire need to develop new widely adaptable cultivars with broad genetic base carrying resistance genes for biotic as well as abiotic stresses. Among the potential resistance sources, rye (Secale cereale) genome is one of important source that has extensively been explored by the 
breeders for bread wheat improvement programme. Wheat-rye substitutions and translocations have been and are frequently used in resistance breeding (Rabinovich 1998) leading to $1 \mathrm{RS} .1 \mathrm{BL}$ translocation in high yielding cultivars currently grown in many parts of the world (Heslop-Harrison et al. 1990). Currently, triticale ( $\times$ Triticosecale) is used as the bridging species for such introgressions due to the low crossability between wheat and rye (Badiyal et al. 2014; Jamwal et al. 2016).

Improvement through conventional breeding approaches is quite lengthy, tedious and require longer duration to reach homozygosity. Conventional plant breeding takes 6 to 7 years to achieve homozygosity in an intervarietal or interspecific hybrid. The doubled haploidy breeding approaches have accelerated the crop improvement efforts by achieving homozygosity in just 2 years. The production of haploids in various crops was initially based on androgenesis and gynogenesis mediated techniques. The genotype specificity and low haploid production efficiency of the techniques limited their use to certain crops like Brassica (Brassica sp.) and rice (Oryza sativa) only. In crops like wheat, barley (Hordeum vulgare) and oats (Avena sativa), these techniques were not well efficient and practicable. The chromosome elimination mediated techniques of haploid induction have revolutionized the genetic improvement programmes of such crops.

As per the report of Chaudhary et al. (2005) and Chaudhary (2008), Imperata cylindrica-mediated chromosome elimination approach of haploid production in wheat was found to be more efficient and superior than maize-mediated system. Kishore et al. (2011) also reported striking success results in respect of induction of haploids in spring and winter wheat $\times$ Himalayan rye derivatives following $I$. cylindrica-mediated system where maize-mediated system failed. With these results in mind, the advantages of doubled haploidy and I. cylindrica, different generations of wheat $\times$ rye and wheat $\times$ triticale were utilized for haploid production following I. cylindrica-mediated chromosome elimination approach.

\section{Materials and Methods}

The present investigation was conducted at the Experimental Farm of the Department of Crop Improvement, CSK HPKV, Palampur, Himachal Pradesh during winter seasons from 2015 to 2018. The experimental material included different advanced generations of triticale $\times$ wheat and wheat $\times$ rye recombinants. Five genotypes of hexaploid triticale (DT-123, DT-126, TL-2900, TL-2908 and TL-9335), four genotypes of Himalayan rye (Karoki rye, shanoor rye, tino rye and triloki rye) were used with various elite bread wheat genotypes to produce different advanced generations. Staggered sowing of different generations of wheat $\times$ rye (Lahaul Local) introgressed lines and triticale wheat (TW) derived lines was done at 15 day intervals from the last week of October to mid January, 2015-2018 at Experimental Farm of the Department of Crop Improvement, CSKHPKV, Palampur. Each line was sown in two rows, $1.5 \mathrm{~m}$ long with $25 \mathrm{~cm}$ row to row spacing, in each sowing. These different rye introgressed lines were used in wide hybridization programme following I. cylindrica-mediated chromosome elimination approach of doubled haploidy breeding. 
For haploid induction, 15-20 spikes from each cross of each generation were pollinated with pollen source I. cylindrica following protocol of Chaudhary et al. (2002 and 2005). The uppermost internodes of the pollinated culms were injected with 2,4-D solution of $250 \mathrm{ppm}$ concentration (Pratap et al. 2005) at $24 \mathrm{hrs}, 48 \mathrm{hrs}$ and $72 \mathrm{hrs}$ after pollination. The injection holes were sealed by using petroleum jelly. Eighteen to twenty days after of pollination, the crossed spikes were harvested from the tiller base and embryo carrying seeds were identified under a light source as per Bains et al. (1998). Tween-20 was used as disinfectant to wash the embryo carrying pseudoseeds. For surface sterilization of pseudoseeds, 0.1 per cent $\mathrm{HgCl}_{2}$ and 0.1 per cent carbendazim fungicide each for 1-2 minutes were used at the time of embryo rescue in Laminar Air Flow Chamber followed by two washing with autoclaved distilled water. Murashige and Skoog (1962) medium supplemented with essential amino acids was used for embryo rescue. Cold treatment at $4{ }^{\circ} \mathrm{C}$ temperature was given to cultured embryos for first 24 hours. After that, they were incubated in dark in the Plant Growth Chamber for about a week at $20 \pm 2{ }^{\circ} \mathrm{C}$ for regeneration till the roots and shoots initiated. The regenerated plantlets were then moved to the other section of the Plant Growth Chamber at $20 \pm 2{ }^{\circ} \mathrm{C}$ with 10/14 hrs light/dark profile with 75 per cent relative humidity for continued plantlet development. Data were recorded with respect of three haploid induction parameters viz., pseudo-seed formation frequency (number of pseudoseeds formed/total number of florets pollinated), embryo formation frequency (number of embryos formed/total number of pseudoseeds formed) and embryo regeneration frequency (number of haploid plantlets developed/total number of embryos cultured). Analysis of data was done by using simple $t$-test.

\section{Results}

\section{Pseudoseed formation frequency}

In case of triticale $\times$ wheat hybridization, range of pseudoseed formation frequency varied from $0-86.84 \%$ in $\mathrm{F}_{2}, 42.86-79.73 \%$ in $\mathrm{BC}_{1} \mathrm{~F}_{1}, 8.14-78.69 \%$ in $\mathrm{BC}_{1} \mathrm{~F}_{2}, 0-78.29 \%$ in $\mathrm{BC}_{1} \mathrm{~F}_{3}, 7.14-78.50 \%$ in $\mathrm{BC}_{1} \mathrm{~F}_{4}$ and $0-88.24 \%$ in $\mathrm{BC}_{1} \mathrm{~F}_{5}$ (Table 1). As per the results, in $\mathrm{F}_{2}$ generation, cross (TL-2908 $\times$ DH-86) $\times$ IC responded for maximum frequency of pseudoseed formation. Whereas in backcross generations viz., $\mathrm{BC}_{1} \mathrm{~F}_{1}, \mathrm{BC}_{1} \mathrm{~F}_{2}, \mathrm{BC}_{1} \mathrm{~F}_{3}, \mathrm{BC}_{1} \mathrm{~F}_{4}$ and $\mathrm{BC}_{1} \mathrm{~F}_{5}$, the crosses namely, (TL-2900 $\left.\times \mathrm{HD}-2380\right) \times \mathrm{HPW}-89 \times \mathrm{IC}$, (TL-2908 $\times \mathrm{DH}-$ $110) \times \mathrm{DH}-100 \times \mathrm{IC},(\mathrm{TL}-2908 \times \mathrm{DH}-86) \times \mathrm{DH}-86 \times \mathrm{IC},(\mathrm{TL}-2900 \times \mathrm{HPW}-236) \times \mathrm{HPW}-$ $236 \times$ IC, $($ TL-2908 $\times$ DH-776) $\times$ DH-776 $\times$ IC, respectively, showed significantly higher pseudoseed formation frequency (Table 1$)$. In wheat $\times$ rye hybridization, range of pseudoseed formation frequency was found to be $0-80.07 \%$ in $\mathrm{F}_{1}, 63.21-81.11 \%$ in $\mathrm{F}_{2}, 61.52-$ $80.85 \%$ in $\mathrm{BC}_{1} \mathrm{~F}_{1}, 51.24-77.80 \%$ in $\mathrm{BC}_{1} \mathrm{~F}_{2}, 59.24-89.87 \%$ in $\mathrm{BC}_{1} \mathrm{~F}_{3}$ and $28.46-80.06 \%$ in $\mathrm{BC}_{1} \mathrm{~F}_{4}$ (Table 2). In $\mathrm{F}_{1}$ and $\mathrm{F}_{2},(\mathrm{DH}-84 \times$ Tino rye) $\times \mathrm{IC}$ and (Badkanku $1 \times \mathrm{Sh}$. Rye) $\times$ IC, respectively were found to exhibit significantly higher pseudoseed formation frequency. Whereas among backcrosses, crosses namely, $(\mathrm{BW}-216 \times \mathrm{Rye}) \times \mathrm{BW}-216 \times \mathrm{IC}$ in $\mathrm{BC}_{1} \mathrm{~F}_{3}$ and $(\mathrm{BW}-215 \times \mathrm{Rye}) \times \mathrm{BW}-215 \times \mathrm{IC} 3 \mathrm{P}\left(1 \mathrm{P}_{1}\right)$ in $\mathrm{BC}_{1} \mathrm{~F}_{4}$ revealed significantly 
Table 1. Frequency of various haploid induction parameters among triticale $\times$ wheat backcrosses and various segregating generations when hybridized with Imperata cylindrica

\begin{tabular}{|c|c|c|c|}
\hline $\mathrm{F}_{2}$ & $\mathrm{PFF}$ & $\mathrm{EFF}$ & ERF \\
\hline$(\mathrm{TL}-2900 \times \mathrm{HPW}-89) \times \mathrm{IC}$ & 21.15 & $9.09 * *$ & $33.33 * *$ \\
\hline$(\mathrm{TL}-2900 \times \mathrm{DH}-5) \times \mathrm{IC}$ & 27.46 & $9.24 * *$ & $29.41 * *$ \\
\hline$(\mathrm{TL}-2900 \times \mathrm{VL}-829) \times \mathrm{IC}$ & 2.94 & 0 & 0 \\
\hline$(\mathrm{TL}-2900 \times \mathrm{VL}-892) \times \mathrm{IC}$ & 0 & 0 & 0 \\
\hline$(\mathrm{TL}-2900 \times \mathrm{HPW}-368) \times \mathrm{IC}$ & 0 & 0 & 0 \\
\hline$(\mathrm{TL}-2900 \times \mathrm{C}-306) \times \mathrm{IC}$ & 1.05 & 0 & 0 \\
\hline$(\mathrm{TL}-2900 \times \mathrm{HPW}-155) \times \mathrm{IC}$ & 0 & 0 & 0 \\
\hline$(\mathrm{TL}-2900 \times \mathrm{DH}-86) \times \mathrm{IC}$ & $65.48 * *$ & $20.00 * *$ & $36.36^{* *}$ \\
\hline$(\mathrm{TL}-2908 \times \mathrm{BW}-215) \times \mathrm{IC}$ & 0 & 0 & 0 \\
\hline$(\mathrm{TL}-2908 \times \mathrm{VL}-892) \times \mathrm{IC}$ & $42.94 * *$ & 0 & 0 \\
\hline$(\mathrm{TL}-2908 \times \mathrm{HPW}-368) \times \mathrm{IC}$ & 0 & 0 & 0 \\
\hline$(\mathrm{TL}-2908 \times \mathrm{DH}-86) \times \mathrm{IC}$ & $86.84 * *$ & 0 & 0 \\
\hline Mean & 20.66 & 3.19 & 8.26 \\
\hline $\mathrm{SE} \pm$ & 8.60 & 1.84 & 4.33 \\
\hline \multicolumn{4}{|c|}{$\mathrm{BC}_{1} \mathrm{~F}_{1}$} \\
\hline$(\mathrm{TL}-2900 \times \mathrm{HD}-2380) \times \mathrm{HPW}-89 \times \mathrm{IC}$ & $79.73 *$ & 24.29 & $2.33 *$ \\
\hline$(\mathrm{TL}-2900 \times \mathrm{VL}-829) \times \mathrm{VL}-829 \times \mathrm{IC}$ & 65.71 & 29.35 & 0 \\
\hline$(\mathrm{TL}-2900 \times \mathrm{C}-306) \times \mathrm{C}-306 \times \mathrm{IC}$ & 42.86 & 27.27 & 0 \\
\hline$(\mathrm{TL}-2900 \times \mathrm{VL}-892) \times \mathrm{VL}-892 \times \mathrm{IC}$ & 75.00 & 29.91 & $2.86^{*}$ \\
\hline$(\mathrm{TL}-2908 \times \mathrm{VL}-892) \times \mathrm{VL}-892 \times \mathrm{IC}$ & 68.45 & 25.22 & 0 \\
\hline$(\mathrm{TL}-2908 \times \mathrm{C}-306) \times \mathrm{C}-306 \times \mathrm{IC}$ & $76.74 *$ & $43.94 * *$ & 0 \\
\hline$(\mathrm{TL}-2908 \times \mathrm{BW}-215) \times \mathrm{C}-306 \times \mathrm{IC}$ & 64.94 & 35.00 & $2.86^{*}$ \\
\hline Mean & 67.63 & 30.71 & 1.15 \\
\hline $\mathbf{S E} \pm$ & 4.65 & 2.58 & 0.55 \\
\hline \multicolumn{4}{|c|}{$\mathrm{BC}_{1} \mathrm{~F}_{2}$} \\
\hline$(\mathrm{DT}-123 \times$ DH-776) $\times$ DH-776 $\times$ IC & 8.14 & 0 & 0 \\
\hline$($ DT-123 $\times$ HS-295) $\times$ HS-295 $\times$ IC & 51.79 & $27.65^{*}$ & 38.84 \\
\hline$(\mathrm{TL}-2908 \times \mathrm{DH}-110) \times \mathrm{DH}-100 \times \mathrm{IC}$ & $78.69 *$ & 9.38 & 33.33 \\
\hline$(\mathrm{TL}-2908 \times \mathrm{DH}-65) \times \mathrm{DH}-86 \times \mathrm{IC}$ & 44.41 & 11.95 & $59.21 *$ \\
\hline$(\mathrm{TL}-2900 \times \mathrm{DH}-776) \times \mathrm{DH}-114 \times \mathrm{IC}$ & 62.62 & 1.98 & 20.00 \\
\hline Mean & 49.13 & 10.19 & 30.28 \\
\hline $\mathrm{SE} \pm$ & 11.76 & 4.90 & 9.85 \\
\hline
\end{tabular}


Table 1 (cont.)

\begin{tabular}{|c|c|c|c|}
\hline $\mathrm{F}_{2}$ & PFF & EFF & ERF \\
\hline \multicolumn{4}{|c|}{$\mathrm{BC}_{1} \mathrm{~F}_{3}$} \\
\hline$(\mathrm{DT}-123 \times \mathrm{VL}-829) \times \mathrm{VL}-829 \times \mathrm{IC}$ & 53.20 & 9.18 & $37.93 *$ \\
\hline$(\mathrm{DT}-123 \times$ DH-776) $\times$ DH-776 $\times$ IC & $62.55^{*}$ & 4.56 & $53.33 * *$ \\
\hline$($ DT-123 $\times$ HS- 295$) \times$ HS-295 $\times$ IC & 55.47 & $26.28 * *$ & $68.06 * *$ \\
\hline$(\mathrm{DT}-123 \times \mathrm{C}-306) \times \mathrm{C}-306 \times \mathrm{IC}$ & 12.21 & 4.41 & 33.33 \\
\hline$(\mathrm{DT}-126 \times \mathrm{DH}-84) \times \mathrm{DH}-84 \times \mathrm{IC}$ & 0 & 0 & 0 \\
\hline$(\mathrm{DT}-126 \times \mathrm{VL}-829) \times \mathrm{VL}-829 \times \mathrm{IC}$ & 0 & 0 & 0 \\
\hline$(\mathrm{DT}-126 \times \mathrm{DH}-776) \times \mathrm{DH}-776 \times \mathrm{IC}$ & 58.60 & 11.01 & 16.67 \\
\hline$\left(\mathrm{TL}-2900 \times \mathrm{C}_{1}-11\right) \times \mathrm{C}_{1}-11 \times \mathrm{IC}$ & $77.23 * *$ & $40.12 * *$ & $42.42 * *$ \\
\hline$(\mathrm{TL}-2900 \times \mathrm{DH}-776) \times \mathrm{DH}-776 \times \mathrm{IC}$ & 49.56 & $16.14 *$ & 31.94 \\
\hline$(\mathrm{TL}-2900 \times \mathrm{DH}-776) \times \mathrm{DH}-114 \times \mathrm{IC}$ & 49.22 & 1.59 & 0 \\
\hline$(\mathrm{TL}-2900 \times \mathrm{HPW}-236) \times \mathrm{HPW}-236 \times \mathrm{IC}$ & $65.68 * *$ & 12.90 & $50.88 * *$ \\
\hline$(\mathrm{TL}-2908 \times \mathrm{DH}-776) \times \mathrm{DH}-65 \times \mathrm{IC}$ & $68.52 * *$ & $25.68 * *$ & $40.35 * *$ \\
\hline$(\mathrm{TL}-2908 \times \mathrm{DH}-776) \times \mathrm{DH}-776 \times \mathrm{IC}$ & 3.20 & $37.50 * *$ & 16.67 \\
\hline$(\mathrm{TL}-2908 \times \mathrm{DH}-86) \times \mathrm{DH}-86 \times \mathrm{IC}$ & $78.29 * *$ & $19.73 * *$ & 31.08 \\
\hline$(\mathrm{TL}-2908 \times \mathrm{DH}-84) \times \mathrm{DH}-84 \times \mathrm{IC}$ & $72.39 * *$ & 1.27 & 0 \\
\hline$(\mathrm{TL}-2908 \times \mathrm{DH}-110) \times \mathrm{DH}-100 \times \mathrm{IC}$ & 52.71 & 6.86 & 16.67 \\
\hline$(\mathrm{TL}-2908 \times \mathrm{DH}-65) \times \mathrm{DH}-86 \times \mathrm{IC}$ & 32.05 & $22.67 * *$ & $35.29 *$ \\
\hline$\left(\mathrm{TL}-9335 \times \mathrm{C}_{1}-11\right) \times \mathrm{C}_{1}-11 \times \mathrm{IC}$ & $64.55^{*}$ & 5.80 & $38.46^{*}$ \\
\hline$(\mathrm{TL}-9335 \times \mathrm{DH}-84) \times \mathrm{DH}-84 \times \mathrm{IC}$ & $72.22 * *$ & $17.19 *$ & 34.21 \\
\hline$(\mathrm{TL}-9335 \times \mathrm{DH}-86) \times \mathrm{DH}-86 \times \mathrm{IC}$ & 54.76 & 10.56 & $35.29 *$ \\
\hline Mean & 49.12 & 13.67 & 29.13 \\
\hline $\mathrm{SE} \pm$ & 5.75 & 2.66 & 4.30 \\
\hline \multicolumn{4}{|c|}{$\mathrm{BC}_{1} \mathrm{~F}_{4}$} \\
\hline$($ DT-123 $\times$ HS-295) $\times$ HS-295 $\times$ IC & 56.64 & $9.01 *$ & $41.67 * *$ \\
\hline$(\mathrm{DT}-123 \times \mathrm{DH}-776) \times \mathrm{DH}-776 \times \mathrm{IC}$ & 52.26 & $17.27 * *$ & $39.58 * *$ \\
\hline$(\mathrm{DT}-123 \times \mathrm{C}-306) \times \mathrm{C}-306 \times \mathrm{IC}$ & 30.00 & 0 & 0 \\
\hline$(\mathrm{DT}-126 \times \mathrm{VL}-829) \times \mathrm{VL}-829 \times \mathrm{IC}$ & 7.14 & 0 & 0 \\
\hline$(\mathrm{DT}-126 \times \mathrm{DH}-84) \times \mathrm{DH}-84 \times \mathrm{IC}$ & 59.38 & $21.05^{* *}$ & 12.50 \\
\hline$(\mathrm{DT}-126 \times$ DH-776) $\times$ DH $-776 \times$ IC & 46.81 & 0 & 0 \\
\hline$($ TL-9335 $\times$ DH-120) $\times$ DH-120 $\times$ IC & $76.56^{* *}$ & $12.59 * *$ & $32.43 * *$ \\
\hline$(\mathrm{TL}-9335 \times \mathrm{DH}-86) \times \mathrm{DH}-86 \times \mathrm{IC}$ & $64.75^{*}$ & $8.11 *$ & $42.86^{* *}$ \\
\hline$(\mathrm{TL}-9335 \times \mathrm{DH}-84) \times \mathrm{DH}-84 \times \mathrm{IC}$ & $77.22 * *$ & 6.47 & $22.22 *$ \\
\hline$(\mathrm{TL}-2900 \times \mathrm{DH}-776) \times \mathrm{DH}-776 \times \mathrm{IC}$ & 15.56 & 0 & 0 \\
\hline
\end{tabular}


Table 1 (cont.)

\begin{tabular}{|c|c|c|c|}
\hline $\mathrm{F}_{2}$ & $\mathrm{PFF}$ & $\mathrm{EFF}$ & ERF \\
\hline$(\mathrm{TL}-2900 \times \mathrm{DH}-776) \times \mathrm{DH}-114 \times \mathrm{IC}$ & $64.72 *$ & 5.83 & $35.00 * *$ \\
\hline$(\mathrm{TL}-2900 \times \mathrm{HPW}-236) \times \mathrm{HPW}-236 \times \mathrm{IC}$ & $78.50 * *$ & $8.28 *$ & $30.77 * *$ \\
\hline$(\mathrm{TL}-2908 \times \mathrm{DH}-65) \times \mathrm{DH}-86 \times \mathrm{IC}$ & 46.95 & $24.00 * *$ & $41.67 * *$ \\
\hline$(\mathrm{TL}-2908 \times \mathrm{DH}-120) \times \mathrm{DH}-120 \times \mathrm{IC}$ & 18.64 & 0 & 0 \\
\hline$(\mathrm{TL}-2908 \times \mathrm{DH}-100) \times \mathrm{DH}-100 \times \mathrm{IC}$ & 62.39 & 5.48 & 12.50 \\
\hline$(\mathrm{TL}-2908 \times \mathrm{DH}-86) \times \mathrm{DH}-86 \times \mathrm{IC}$ & 61.96 & 0.88 & 0 \\
\hline$(\mathrm{TL}-2908 \times \mathrm{HPW}-236) \times \mathrm{HPW}-236 \times \mathrm{IC}$ & $76.25^{*}$ & 0 & 0 \\
\hline$(\mathrm{TL}-2908 \times \mathrm{DH}-776) \times \mathrm{DH}-65 \times \mathrm{IC}$ & 61.25 & 7.14 & 14.29 \\
\hline Mean & 53.17 & 7.01 & 18.08 \\
\hline $\mathbf{S E} \pm$ & 5.17 & 1.78 & 4.13 \\
\hline \multicolumn{4}{|c|}{$\mathrm{BC}_{1} \mathrm{~F}_{5}$} \\
\hline$(\mathrm{DT}-123 \times \mathrm{DH}-776) \times \mathrm{DH}-776 \times \mathrm{IC}$ & 65.05 & 3.36 & 22.22 \\
\hline$(\mathrm{DT}-123 \times$ HS-295) $\times$ HS-295 $\times$ IC & 63.07 & $15.94 * *$ & $30.00 * *$ \\
\hline$(\mathrm{DT}-123 \times \mathrm{C}-306) \times \mathrm{C}-306 \times \mathrm{IC}$ & 33.65 & 0 & 0 \\
\hline$(\mathrm{DT}-126 \times \mathrm{DH}-84) \times \mathrm{DH}-84 \times \mathrm{IC}$ & 0 & 0 & 0 \\
\hline$(\mathrm{DT}-126 \times \mathrm{DH}-776) \times \mathrm{DH}-776 \times \mathrm{IC}$ & 59.16 & 8.79 & $42.86^{* *}$ \\
\hline$(\mathrm{DT}-126 \times \mathrm{VL}-829) \times \mathrm{VL}-829 \times \mathrm{IC}$ & 30.23 & 0 & 0 \\
\hline$(\mathrm{TL}-2900 \times \mathrm{DH}-776) \times \mathrm{DH}-776 \times \mathrm{IC}$ & 60.14 & $13.48 *$ & 12.50 \\
\hline$(\mathrm{TL}-2900 \times \mathrm{HPW}-236) \times \mathrm{HPW}-236 \times \mathrm{IC}$ & $84.46^{* *}$ & 4.00 & 20.00 \\
\hline$(\mathrm{TL}-2908 \times \mathrm{DH}-120) \times \mathrm{DH}-120 \times \mathrm{IC}$ & $76.32 *$ & $13.79 *$ & $33.33 * *$ \\
\hline$(\mathrm{TL}-2908 \times \mathrm{DH}-776) \times \mathrm{DH}-776 \times \mathrm{IC}$ & $88.24 * *$ & $36.67 * *$ & 18.18 \\
\hline$(\mathrm{TL}-9335 \times \mathrm{DH}-84) \times \mathrm{DH}-84 \times \mathrm{IC}$ & 30.88 & 4.76 & 0 \\
\hline$(\mathrm{TL}-9335 \times$ DH-120) $\times$ DH-120 $\times$ IC & $73.36^{*}$ & $12.10 *$ & 21.05 \\
\hline$(\mathrm{TL}-9335 \times \mathrm{DH}-86) \times \mathrm{DH}-86 \times \mathrm{IC}$ & $82.12 * *$ & 8.49 & $34.78 * *$ \\
\hline Mean & 57.44 & 9.34 & 18.07 \\
\hline $\mathbf{S E} \pm$ & 7.30 & 2.75 & 4.11 \\
\hline
\end{tabular}

*Significant at $\mathrm{P} \leq 0.05$ and $* *$ Significant at $\mathrm{P} \leq 0.01 ; \mathrm{PFF}=$ pseudoseed formation frequency; $\mathrm{EFF}=$ embryo formation frequency; $\mathrm{ERF}=$ embryo regeneration frequency; $\mathrm{KR}=$ Karoki Rye; Sh. rye = Shanoor rye; $\mathrm{IC}=$ Imperata cylindrica .

Table 2. Frequency of various haploid induction parameters among wheat $\times$ rye backcrosses and various segregating generations when hybridized with Imperata cylindrica

\begin{tabular}{|l|c|c|c|}
\hline \multicolumn{1}{|c|}{$\mathrm{F}_{1}$} & PFF & EFF & ERF \\
\hline$(\mathrm{HPW}-368 \times \mathrm{KR}) \times \mathrm{IC}$ & 2.27 & 0 & 0 \\
\hline$(\mathrm{HPW}-368 \times$ Triloki rye $) \times \mathrm{IC}$ & 60.55 & 7.51 & $48.00^{* *}$ \\
\hline$(\mathrm{C}-306 \times \mathrm{KR}) \times \mathrm{IC}$ & 0 & 0 & 0 \\
\hline
\end{tabular}


Table 2 (cont.)

\begin{tabular}{|c|c|c|c|}
\hline $\mathrm{F}_{1}$ & PFF & EFF & ERF \\
\hline$($ VL- $892 \times$ Sh. Rye $) \times$ IC & $77.27 *$ & $23.53 * *$ & $50.00 * *$ \\
\hline$(\mathrm{VL}-829 \times \mathrm{KR}) \times \mathrm{IC}$ & $69.48 *$ & $22.18 * *$ & $50.94 * *$ \\
\hline$($ Badkanku $1 \times$ Sh . Rye $) \times$ IC & $70.68^{*}$ & 3.33 & 22.22 \\
\hline$\left(\mathrm{C}_{1}-1 \times \mathrm{KR}\right) \times \mathrm{IC}$ & 0 & 0 & 0 \\
\hline$(\mathrm{HS}-295 \times \mathrm{KR}) \times \mathrm{IC}$ & $80.07 * *$ & $20.33 * *$ & $45.92 * *$ \\
\hline$(\mathrm{DH}-84 \times$ Tino rye $) \times \mathrm{IC}$ & $78.77 * *$ & $38.70 * *$ & $33.71 *$ \\
\hline$($ DH $-40 \times$ Tino rye $) \times$ IC & $77.38 *$ & 0 & 0 \\
\hline$(\mathrm{DH}-84 \times \mathrm{KR}) \times \mathrm{IC}$ & 7.05 & 0 & 0 \\
\hline Mean & 47.59 & 10.51 & 22.80 \\
\hline $\mathbf{S E} \pm$ & 10.96 & 4.06 & 7.02 \\
\hline \multicolumn{4}{|c|}{$\mathrm{F}_{2}$} \\
\hline$($ HPW-368 $\times$ Triloki rye $) \times$ IC & 75.17 & 15.63 & 34.29 \\
\hline$(\mathrm{C}-306 \times \mathrm{KR}) \times \mathrm{IC}$ & 63.21 & 14.93 & 30.00 \\
\hline$(\mathrm{HPW}-89 \times \mathrm{KR}) \times \mathrm{IC}$ & 69.47 & 2.20 & 25.00 \\
\hline$(\mathrm{HS}-295 \times \mathrm{KR}) \times \mathrm{IC}$ & $80.39 * *$ & $20.73 *$ & 29.41 \\
\hline$(\mathrm{VL}-829 \times \mathrm{KR}) \times \mathrm{IC}$ & 74.65 & $16.51^{*}$ & 42.86 \\
\hline$($ Badkanku $1 \times$ Sh. Rye $) \times$ IC & $81.11 * *$ & 0 & 0 \\
\hline$($ VL- $892 \times$ Sh. Rye $) \times$ IC & 69.77 & 15.00 & $77.78^{*}$ \\
\hline$(\mathrm{DH}-84 \times$ Tino rye $) \times \mathrm{IC}$ & 68.23 & 14.50 & 31.58 \\
\hline$(\mathrm{DH}-40 \times$ Tino rye $) \times \mathrm{IC}$ & 75.56 & 0 & 0 \\
\hline Mean & 73.06 & 11.05 & 30.10 \\
\hline $\mathrm{SE} \pm$ & 1.96 & 2.66 & 7.72 \\
\hline \multicolumn{4}{|c|}{$\mathrm{BC}_{1} \mathrm{~F}_{1}$} \\
\hline$(\mathrm{HS}-295 \times \mathrm{KR}) \times \mathrm{HS}-295 \times \mathrm{IC}$ & 76.62 & 24.35 & $36.27 *$ \\
\hline$(\mathrm{HPW}-89 \times \mathrm{KR}) \times \mathrm{HPW}-89 \times \mathrm{IC}$ & 67.63 & 9.50 & 28.75 \\
\hline$(\mathrm{VL}-829 \times \mathrm{KR}) \times \mathrm{VL}-892 \times \mathrm{IC}$ & 61.52 & 17.54 & 24.32 \\
\hline$(\mathrm{VL}-829 \times \mathrm{KR}) \times \mathrm{HPW}-349 \times \mathrm{IC}$ & 80.85 & 27.98 & 27.84 \\
\hline Mean & 71.65 & 19.84 & 29.30 \\
\hline $\mathbf{S E} \pm$ & 4.36 & 4.07 & 2.51 \\
\hline \multicolumn{4}{|c|}{$\mathrm{BC}_{1} \mathrm{~F}_{2}$} \\
\hline$(\mathrm{HS}-295 \times \mathrm{KR}) \times \mathrm{HS}-295 \times \mathrm{IC}$ & 73.09 & 16.78 & 27.27 \\
\hline$(\mathrm{HPW}-89 \times \mathrm{KR}) \times \mathrm{HPW}-89 \times \mathrm{IC}$ & 51.24 & 12.73 & 33.33 \\
\hline$(\mathrm{VL}-829 \times \mathrm{KR}) \times \mathrm{VL}-892 \times \mathrm{IC}$ & 77.80 & 5.75 & 32.14 \\
\hline$(\mathrm{VL}-829 \times \mathrm{KR}) \times \mathrm{HPW}-349 \times \mathrm{IC}$ & 75.28 & 8.40 & $41.18 *$ \\
\hline
\end{tabular}


Table 2 (cont.)

\begin{tabular}{|c|c|c|c|}
\hline $\mathrm{F}_{1}$ & PFF & EFF & ERF \\
\hline$(\mathrm{C}-306 \times \mathrm{KR}) \times \mathrm{C}-306 \times \mathrm{IC}$ & 61.45 & 16.77 & $40.74 *$ \\
\hline Mean & 67.77 & 12.08 & 34.93 \\
\hline $\mathbf{S E} \pm$ & 4.99 & 2.21 & 2.66 \\
\hline \multicolumn{4}{|c|}{$\mathrm{BC}_{1} \mathrm{~F}_{3}$} \\
\hline$(\mathrm{HPW}-147 \times$ Rye $) \times \mathrm{HPW}-147 \times \mathrm{IC}$ & $85.96 * *$ & 7.15 & $45.00 *$ \\
\hline$($ BW-216 $\times$ Rye $) \times$ BW $-216 \times$ IC & $89.87 * *$ & 4.36 & 33.33 \\
\hline$(\mathrm{HPW}-89 \times$ Rye $) \times \mathrm{HPW}-89 \times \mathrm{IC}$ & 70.14 & 14.64 & $40.79 *$ \\
\hline$($ VL-892 × Rye $) \times$ VL-892 × IC & $80.38 *$ & 0 & 0 \\
\hline$(\mathrm{HPW}-147 \times$ Rye $) \times \mathrm{HPW}-147 \times \mathrm{IC}(2 \mathrm{P})$ & $81.09 *$ & $25.91 *$ & 26.00 \\
\hline$($ HPW-147 × Rye $) \times$ HPW-147 × IC (3P) & 59.24 & 17.95 & 23.81 \\
\hline$($ HPW-147 × Rye $) \times$ HPW-147 × IC (4P) & 65.96 & $30.65 *$ & 36.84 \\
\hline$($ HPW $-89 \times$ Rye $) \times$ HPW $-89 \times$ IC $(2 P)$ & 43.24 & $65.63 * *$ & $42.86 *$ \\
\hline$(\mathrm{HS}-295 \times \mathrm{KR}) \times \mathrm{HS}-295 \times \mathrm{IC}$ & 62.63 & 17.65 & 28.57 \\
\hline$(\mathrm{VL}-829 \times \mathrm{KR}) \times \mathrm{VL}-892 \times \mathrm{IC}$ & 76.41 & 3.69 & 12.50 \\
\hline$(\mathrm{VL}-829 \times \mathrm{KR}) \times \mathrm{HPW}-349 \times \mathrm{IC}$ & 76.87 & 10.18 & $47.83 * *$ \\
\hline Mean & 71.98 & 17.98 & 30.68 \\
\hline $\mathrm{SE} \pm$ & 4.08 & 5.56 & 4.41 \\
\hline \multicolumn{4}{|c|}{$\mathrm{BC}_{1} \mathrm{~F}_{4}$} \\
\hline$(\mathrm{HPW}-89 \times$ Rye $) \times \mathrm{HPW}-89 \times$ IC & $73.97 * *$ & 1.68 & 33.33 \\
\hline$($ BW-215 $\times$ Rye $) \times$ BW-215 $\times$ IC & $74.68 * *$ & 1.27 & 33.33 \\
\hline$(\mathrm{HPW}-89 \times$ Rye $) \times \mathrm{HPW}-89 \times \mathrm{IC} 3 \mathrm{P}\left(1 \mathrm{P}_{1}\right)$ & $74.48 * *$ & 1.73 & $40.00 * *$ \\
\hline$(\mathrm{HPW}-89 \times$ Rye $) \times \mathrm{HPW}-89 \times \mathrm{IC} 3 \mathrm{P}\left(3 \mathrm{P}_{1}\right)$ & $79.77 * *$ & 1.99 & $57.14 * *$ \\
\hline$(\mathrm{HPW}-89 \times$ Rye $) \times \mathrm{HPW}-89 \times \mathrm{IC} 1 \mathrm{P}\left(2 \mathrm{P}_{1}\right)$ & $69.63 *$ & 2.68 & $37.50 *$ \\
\hline$(\mathrm{HPW}-89 \times$ Rye $) \times \mathrm{HPW}-89 \times \mathrm{IC} 2 \mathrm{P}\left(3 \mathrm{P}_{1}\right)$ & 64.41 & 2.89 & $45.45^{* *}$ \\
\hline$(\mathrm{HPW}-89 \times$ Rye $) \times \mathrm{HPW}-89 \times \mathrm{IC} 2 \mathrm{P}\left(1 \mathrm{P}_{1}\right)$ & 64.77 & 2.20 & 28.57 \\
\hline$(\mathrm{HPW}-89 \times$ Rye $) \times \mathrm{HPW}-89 \times \mathrm{IC} 3 \mathrm{P}\left(2 \mathrm{P}_{1}\right)$ & 51.85 & 3.06 & 33.33 \\
\hline$(\mathrm{HPW}-89 \times$ Rye $) \times \mathrm{HPW}-89 \times \mathrm{IC} 1 \mathrm{P}\left(1 \mathrm{P}_{1}\right)$ & 40.63 & 0.77 & 0 \\
\hline$(\mathrm{HPW}-89 \times$ Rye $) \times \mathrm{HPW}-89 \times \mathrm{IC} 2 \mathrm{P}\left(2 \mathrm{P}_{1}\right)$ & 28.46 & 2.70 & 0 \\
\hline$(\mathrm{BW}-215 \times$ Rye $) \times \mathrm{BW}-215 \times \mathrm{IC} 2 \mathrm{P}\left(3 \mathrm{P}_{1}\right)$ & $66.96 *$ & 0.27 & 0 \\
\hline$(\mathrm{BW}-215 \times$ Rye $) \times \mathrm{BW}-215 \times \mathrm{IC} 2 \mathrm{P}\left(1 \mathrm{P}_{1}\right)$ & $72.54 * *$ & 1.59 & 25.00 \\
\hline$(\mathrm{BW}-215 \times$ Rye $) \times \mathrm{BW}-215 \times \mathrm{IC} 3 \mathrm{P}\left(1 \mathrm{P}_{1}\right)$ & $80.06 * *$ & $29.05 * *$ & $53.06 * *$ \\
\hline$(\mathrm{BW}-215 \times$ Rye $) \times \mathrm{BW}-215 \times \mathrm{IC} 4 \mathrm{P}\left(1 \mathrm{P}_{1}\right)$ & 45.95 & $47.06^{* *}$ & $43.75^{* *}$ \\
\hline$($ BW-215 × Rye $) \times$ BW-215 × IC $(1 P)$ & 44.27 & 3.53 & 33.33 \\
\hline$(\mathrm{BW}-215 \times$ Rye $) \times \mathrm{BW}-215 \times \mathrm{IC} 3 \mathrm{P}\left(2 \mathrm{P}_{1}\right)$ & 59.29 & $22.89 * *$ & $42.11 * *$ \\
\hline
\end{tabular}


Table 2 (cont.)

\begin{tabular}{|l|c|c|c|}
\hline \multicolumn{1}{|c|}{$\mathrm{F}_{1}$} & PFF & EFF & ERF \\
\hline$(\mathrm{BW}-215 \times$ Rye $) \times \mathrm{BW}-215 \times \mathrm{IC} 3 \mathrm{P}\left(3 \mathrm{P}_{1}\right)$ & 41.53 & 4.08 & 0 \\
\hline$(\mathrm{BW}-215 \times$ Rye $) \times \mathrm{BW}-215 \times \mathrm{IC} 2 \mathrm{P}\left(2 \mathrm{P}_{1}\right)$ & 35.61 & 0 & 0 \\
\hline Mean & $\mathbf{5 9 . 3 8}$ & $\mathbf{7 . 1 9}$ & $\mathbf{2 8 . 1 1}$ \\
\hline SE \pm & $\mathbf{3 . 8 6}$ & $\mathbf{2 . 9 9}$ & $\mathbf{4 . 6 1}$ \\
\hline
\end{tabular}

*Significant at $\mathrm{P} \leq 0.05$ and $* *$ Significant at $\mathrm{P} \leq 0.01$; $\mathrm{PFF}=$ pseudoseed formation frequency; $\mathrm{EFF}=$ embryo formation frequency; $\mathrm{ERF}=$ embryo regeneration frequency; $\mathrm{KR}=$ Karoki Rye; Sh. rye = Shanoor rye; IC = Imperata cylindrica .

Table 3. Mean performance of different generations in triticale $\times$ wheat and wheat $\times$ rye hybridization towards haploid induction parameters

\begin{tabular}{|l|c|c|c|c|c|c|}
\hline \multirow{2}{*}{ Generations } & \multicolumn{3}{|c|}{ Triticale $\times$ wheat } & \multicolumn{3}{c|}{ Wheat $\times$ rye } \\
\cline { 2 - 7 } & PFF & EFF & ERF & PFF & EFF & ERF \\
\hline $\mathrm{F}_{1}$ & - & - & - & 47.59 & 10.51 & 22.80 \\
\hline $\mathrm{F}_{2}$ & 20.66 & 3.19 & 8.26 & 73.06 & 11.05 & 30.10 \\
\hline $\mathrm{BC}_{1} \mathrm{~F}_{1}$ & $67.63^{*}$ & $30.71^{* *}$ & 1.15 & 71.65 & $19.84^{*}$ & 29.30 \\
\hline $\mathrm{BC}_{1} \mathrm{~F}_{2}$ & 49.13 & 10.19 & $30.28^{*}$ & 67.77 & 12.08 & $34.93^{* *}$ \\
\hline $\mathrm{BC}_{1} \mathrm{~F}_{3}$ & 49.12 & 13.67 & $29.13^{*}$ & 71.98 & $17.98^{*}$ & 30.68 \\
\hline $\mathrm{BC}_{1} \mathrm{~F}_{4}$ & 53.17 & 7.01 & 18.08 & 59.38 & 7.19 & 28.11 \\
\hline $\mathrm{BC}_{1} \mathrm{~F}_{5}$ & 57.44 & 9.34 & 18.07 & - & - & - \\
\hline $\mathrm{Mean}$ & $\mathbf{4 9 . 5 2}$ & $\mathbf{1 2 . 3 5}$ & $\mathbf{1 7 . 4 9}$ & $\mathbf{6 5 . 2 4}$ & $\mathbf{1 3 . 1 1}$ & $\mathbf{2 9 . 3 2}$ \\
\hline $\mathrm{SE} \pm$ & $\mathbf{6 . 4 2}$ & $\mathbf{3 . 9 4}$ & $\mathbf{4 . 6 6}$ & $\mathbf{4 . 0 8}$ & $\mathbf{1 . 9 7}$ & $\mathbf{1 . 6 1}$ \\
\hline
\end{tabular}

*Significant at $\mathrm{P} \leq 0.05$ and $* *$ Significant at $\mathrm{P} \leq 0.01 ; \mathrm{PFF}=$ pseudoseed formation frequency; $\mathrm{EFF}=$ embryo formation frequency; $\mathrm{ERF}=$ embryo regeneration frequency.

higher pseudoseed formation frequency (Table 2). In $\mathrm{BC}_{1} \mathrm{~F}_{1}$ and $\mathrm{BC}_{1} \mathrm{~F}_{2}$ no cross was found to be significantly superior for pseudoseed formation frequency.

Among the different generations in triticale $\times$ wheat hybridization, the results analyzed following Student's $t$-test indicated that mean performance of $\mathrm{BC}_{1} \mathrm{~F}_{1}(67.63 \%)$ was found to be significantly positive and highest for pseudoseed formation frequency (Table 3 ). Whereas in wheat $\times$ rye hybridization, as per results, no generation was found significantly superior towards pseudoseed formation frequency (Table 3 ).

\section{Embryo formation frequency}

In triticale $\times$ wheat hybridization, the range of embryo formation frequency was from 0 to $20.00 \%, 24.29$ to $43.94 \%, 0$ to $27.65 \%, 0$ to $40.12 \%, 0$ to $24.00 \%$ and 0 to $36.67 \%$ in $\mathrm{F}_{2}$, $\mathrm{BC}_{1} \mathrm{~F}_{1}, \mathrm{BC}_{1} \mathrm{~F}_{2}, \mathrm{BC}_{1} \mathrm{~F}_{3}, \mathrm{BC}_{1} \mathrm{~F}_{4}$ and $\mathrm{BC}_{1} \mathrm{~F}_{5}$ generation, respectively (Table 1). In $\mathrm{F}_{2}$ generation, among the various crosses, cross namely, (TL-2900 $\times$ DH-86) $\times$ IC showed significantly higher response towards embryo formation frequency. In backcross generations, 
crosses viz., (TL-2908 $\times$ C-306) $\times$ C-306 $\times$ IC, $($ DT-123 $\times$ HS-295) $\times$ HS-295 $\times$ IC, $($ TL$\left.2900 \times \mathrm{C}_{1}-11\right) \times \mathrm{C}_{1}-11 \times \mathrm{IC}, \quad(\mathrm{TL}-2908 \times \mathrm{DH}-65) \times \mathrm{DH}-86 \times \mathrm{IC}$ and $(\mathrm{TL}-2908 \times \mathrm{DH}-$ 776) $\times \mathrm{DH}-776 \times \mathrm{IC}$ were found to have significantly higher embryo formation frequency in $\mathrm{BC}_{1} \mathrm{~F}_{1}, \mathrm{BC}_{1} \mathrm{~F}_{2}, \mathrm{BC}_{1} \mathrm{~F}_{3}, \mathrm{BC}_{1} \mathrm{~F}_{4}$ and $\mathrm{BC}_{1} \mathrm{~F}_{5}$, respectively (Table 1 ). In wheat $\times$ rye hybridization, range of embryo formation frequency was found to be $0-38.70 \%$ in $\mathrm{F}_{1}$, $0-20.73 \%$ in $\mathrm{F}_{2}, 9.50-27.98 \%$ in $\mathrm{BC}_{1} \mathrm{~F}_{1}, 5.75-16.78 \%$ in $\mathrm{BC}_{1} \mathrm{~F}_{2}, 0-65.63 \%$ in $\mathrm{BC}_{1} \mathrm{~F}_{3}$ and $0-47.06 \%$ in $\mathrm{BC}_{1} \mathrm{~F}_{4}$ (Table 2). The results revealed that crosses namely, (DH-84 $\times$ Tino rye $) \times \mathrm{IC}, \quad(\mathrm{HS}-295 \times \mathrm{KR}) \times \mathrm{IC}, \quad(\mathrm{HPW}-89 \times \mathrm{Rye}) \times \mathrm{HPW}-89 \times \mathrm{IC} \quad(2 \mathrm{P})$ and $(\mathrm{BW}-$ $215 \times$ Rye $) \times \mathrm{BW}-215 \times \mathrm{IC} 4 \mathrm{P}\left(1 \mathrm{P}_{1}\right)$ exhibited significantly higher embryo formation frequency over all the crosses in $\mathrm{F}_{1}, \mathrm{~F}_{2}, \mathrm{BC}_{1} \mathrm{~F}_{3}$ and $\mathrm{BC}_{1} \mathrm{~F}_{4}$, respectively (Table 2). In $\mathrm{BC}_{1} \mathrm{~F}_{1}$ and $\mathrm{BC}_{1} \mathrm{~F}_{2}$ like pseudoseed formation frequency, no cross was found to be significantly superior over others crosses.

In triticale $\times$ wheat hybridization, the analysis of results revealed that $\mathrm{BC}_{1} \mathrm{~F}_{1}(30.71 \%)$ generation had the highest and significantly positive response towards embryo formation frequency over all the generations (Table 3 ). In case of wheat $\times$ rye hybridization, among the different generations, mean performance of $\mathrm{BC}_{1} \mathrm{~F}_{1}(19.84 \%)$ and $\mathrm{BC}_{1} \mathrm{~F}_{3}(17.98 \%)$ were found to exhibit significantly positive and highest response towards embryo formation frequency (Table 3).

\section{Embryo regeneration frequency}

In triticale $\times$ wheat hybridization, it ranged from 0 to $36.36 \%$ in $\mathrm{F}_{2}, 0$ to $2.86 \%$ in $\mathrm{BC}_{1} \mathrm{~F}_{1}$, 0 to $59.21 \%$ in $\mathrm{BC}_{1} \mathrm{~F}_{2}, 0$ to $68.06 \%$ in $\mathrm{BC}_{1} \mathrm{~F}_{3}, 0$ to $42.86 \%$ in $\mathrm{BC}_{1} \mathrm{~F}_{4}$ and 0 to $42.86 \%$ in $\mathrm{BC}_{1} \mathrm{~F}_{5}$ generation (Table 1). The crosses that showed significant highest embryo regeneration frequency in $\mathrm{F}_{2}, \mathrm{BC}_{1} \mathrm{~F}_{1}, \mathrm{BC}_{1} \mathrm{~F}_{2}, \mathrm{BC}_{1} \mathrm{~F}_{3}, \mathrm{BC}_{1} \mathrm{~F}_{4}$ and $\mathrm{BC}_{1} \mathrm{~F}_{5}$ generation were $(\mathrm{TL}-2900 \times \mathrm{DH}-86) \times \mathrm{IC}, \quad(\mathrm{TL}-2900 \times \mathrm{VL}-892) \times \mathrm{VL}-892 \times \mathrm{IC} \quad(\mathrm{TL}-2908 \times \mathrm{BW}-$ $215) \times \mathrm{C}-306 \times \mathrm{IC}, \quad(\mathrm{TL}-2908 \times \mathrm{DH}-65) \times \mathrm{DH}-86 \times \mathrm{IC}, \quad(\mathrm{DT}-123 \times \mathrm{HS}-295) \times \mathrm{HS}-$ $295 \times$ IC, $($ TL-9335 $\times$ DH-86) $\times$ DH-86 $\times$ IC and $($ DT-126 $\times$ DH-776) $\times$ DH-776 $\times$ IC, respectively (Table 1 ). In wheat $\times$ rye hybridization, range of embryo regeneration frequency varied from 0 to $50.94 \%$ in $\mathrm{F}_{1}, 0$ to $77.78 \%$ in $\mathrm{F}_{2}, 24.32$ to $36.27 \%$ in $\mathrm{BC}_{1} \mathrm{~F}_{1}, 27.27$ to $41.18 \%$ in $\mathrm{BC}_{1} \mathrm{~F}_{2}, 0$ to $47.83 \%$ in $\mathrm{BC}_{1} \mathrm{~F}_{3}$ and 0 to $57.14 \%$ in $\mathrm{BC}_{1} \mathrm{~F}_{4}$ generation (Table 2). As per the results, crosses namely, (VL-829 $\times$ KR) $\times$ IC, $($ VL- $892 \times$ Sh. Rye $) \times$ IC, (HS$295 \times \mathrm{KR}) \times \mathrm{HS}-295 \times \mathrm{IC}, \quad(\mathrm{VL}-829 \times \mathrm{KR}) \times \mathrm{HPW}-349 \times \mathrm{IC}, \quad(\mathrm{VL}-829 \times \mathrm{KR}) \times \mathrm{HPW}-$ $349 \times \mathrm{IC}$ and $(\mathrm{HPW}-89 \times \mathrm{Rye}) \times \mathrm{HPW}-89 \times \mathrm{IC} 3 \mathrm{P}\left(3 \mathrm{P}_{1}\right)$ were found to reveal significant maximum embryo regeneration frequency in $\mathrm{F}_{1}, \mathrm{~F}_{2}, \mathrm{BC}_{1} \mathrm{~F}_{1}, \mathrm{BC}_{1} \mathrm{~F}_{2}, \mathrm{BC}_{1} \mathrm{~F}_{3}$ and $\mathrm{BC}_{1} \mathrm{~F}_{4}$ generation, respectively (Table 2 ). Significantly positive embryo regeneration was also observed in the crosses with less embryo formation. It was due to the lower number of embryos.

In case of embryo regeneration frequency, significantly higher response was observed in $\mathrm{BC}_{1} \mathrm{~F}_{2}(30.28 \%)$ and $\mathrm{BC}_{1} \mathrm{~F}_{3}(29.13 \%)$ generations in triticale $\times$ wheat hybridization (Table 3 ). Whereas in wheat $\times$ rye hybridization, analysis of results following Student's $t$-test revealed that among all the generations, $\mathrm{BC}_{1} \mathrm{~F}_{2}(34.93 \%)$ exhibited significantly higher response towards embryo regeneration frequency (Table 3 ). 


\section{Discussion}

In the present investigation, the recombinants and other advanced generations of triticale $\times$ wheat and wheat $\times$ rye hybrids were subjected to $I$. cylindrica-mediated chromosome elimination approach of doubled haploidy breeding at CSKHPKV, Palampur for instantaneous fixation of novel rye genes in the wheat background. Different genotypes in subsequent generations in both triticale $\times$ wheat and wheat $\times$ rye recombinants were found to exhibit variable response towards haploid induction parameters. The reason for such a large variation in pseudoseed formation lies in auxin $(2,4-\mathrm{D})$ application that results in ovary growth (Suenaga 1994). Pratap and Chaudhary (2012) also revealed the influence of auxins on haploid induction parameters. Auxins were reported to cause the enlargement of ovaries even in the absence of embryos (Wedzony et al. 1998). Additionally, high frequencies of pseudoseed formation in different crosses also revealed the genotypic non-specific nature of this parameter (Inagaki and Tahir 1990; Suenaga 1994; Kishore et al. 2011). In case of embryo formation frequency, one of the reasons for such a wide variation is environmental conditions. Many workers across the world depicted the influence of environmental conditions such as temperature, relative humidity and photoperiod on the development of haploid embryos (Campbell et al. 1998; Silva et al. 2002; Ballesteros et al. 2003). Genome/genotypic specificity also plays a key role in haploid induction through I. cylindrica- mediated chromosome elimination approach as suggested by Mukai et al. (2015). In the present study, different genotypes were utilized in various generations for haploid production that resulted in large variation for embryo formation frequency. Mukai et al. (2015) also reported increased crossability of D genome chromosomes (specially 7D) substituted lines in wheat genetic background with $I$. cylindrica for the haploid induction. So, instability in the number of D and R chromosomes can also be attributed for causing variation in haploid embryo formation frequency. The efficiency of haploid embryo regeneration is highly influenced by embryo formation frequency. Wide variation in haploid embryo regeneration can be attributed to low number of embryos formed, in vitro cultural conditions, size and age of embryo to be cultured.

Mean performance of different generations namely, $\mathrm{F}_{1}, \mathrm{~F}_{2}, \mathrm{BC}_{1} \mathrm{~F}_{1}, \mathrm{BC}_{1} \mathrm{~F}_{2}, \mathrm{BC}_{1} \mathrm{~F}_{3}$, $\mathrm{BC}_{1} \mathrm{~F}_{4}$ and $\mathrm{BC}_{1} \mathrm{~F}_{5}$ also revealed significant genetic variability for the three haploid induction parameters. Among all the generations, backcross generations viz., $\mathrm{BC}_{1} \mathrm{~F}_{1}$ (pseudoseed and embryo formation frequency) and $\mathrm{BC}_{1} \mathrm{~F}_{2}$ (embryo regeneration frequency) were found to be significantly higher for haploid induction parameters in both triticale $\times$ wheat and wheat $\times$ rye hybridization. The present findings are in concordance with the earlier report of Kishore et al. (2011) who reported maximum haploid embryos in $\mathrm{BC}_{1} \mathrm{~F}_{1}$ as compared to $\mathrm{BC}_{1} \mathrm{~F}_{2}$ in wheat $\times$ rye derivatives. The encouraging results of backcross generations may be due to the higher wheat genetic content and stability towards higher number of D chromosomes. However further studies are necessary to demonstrate the relationship between backcross generations and haploid induction. 


\section{Acknowledgements}

The authors are thankful to the Department of Science \& Technology for providing financial assistance and CSKHPKV for providing experimental material and fields in the Department of Crop Improvement.

\section{References}

Badiyal, A., Chaudhary, H.K., Jamwal, N.S., Hussain, W., Mahato, A., Bhatt, A.K. 2014. Interactive genotypic influence of triticale and wheat on their crossability and haploid induction under varied agroclimatic regimes. Cereal Res. Commun. 42:700-709.

Bains, N.S., Mangat, G.S., Singh, K., Nanda, G.S. 1998. A simple technique for the identification of embryo carrying seeds from wheat $\times$ maize crosses prior to dissection. Plant Breeding 117:191-192.

Ballesteros, J., García-Llamas, C., Ramirez, M.C., Martín, A., Weber, W.E. 2003. Low relative humidity increases haploid production in durum wheat $\times$ maize crosses. Plant Breeding 122:276-278.

Campbell, A.W., Griffin, W.B., Conner, A.J., Rowarth, J.S., Burritt, D.J. 1998. The effects of temperature and light intensity on embryo numbers in wheat doubled haploid production through wheat $\times$ maize crosses. Ann. Bot-London 82:29-33.

Chaudhary, H.K. 2008. Dynamics of wheat $\times$ Imperata cylindrica - a new chromosome elimination mediated system for efficient haploid induction in wheat. In: Proceedings of the $11^{\text {th }}$ International Wheat Genetics Symposium R. Appels et al. (eds). University of Sydney Press, Sydney, Australia. pp. 647-650.

Chaudhary, H.K., Sethi, G.S., Singh, S., Pratap, A., Sharma, S. 2005. Efficient haploid induction in wheat by using pollen of Imperata cylindrica. Plant Breeding 124:96-98.

Chaudhary, H.K., Singh, S., Sethi, G.S. 2002. Interactive influence of wheat and maize genotypes on haploid induction in winter $\times$ spring wheat hybrids. J. Genet. Breed. 56:259-266.

Heslop-Harrison, J.S., Leitch, A.R., Schwanzacher, T., Anamthawat-Jonsson, K. 1990. Detection and characterization of 1BL/1R translocation in hexaploid wheat. Heredity 65:385-392.

Inagaki, M., Tahir, M. 1990. Comparison of haploid production frequencies in wheat varieties crossed with Hordeum bulbosum L. and maize. Jpn. J. Breed. 40:209-216.

Jamwal, N.S., Chaudhary, H.K., Badiyal, A., Hussain, W. 2016. Factors influencing crossability among triticale and wheat and its subsequent effect along with hybrid necrosis on haploid induction. Acta Agriculturae Scandinavica, Section B-Plant Soil Science 66:282-289.

Kishore, N., Chaudhary, H.K., Chahota, R.K., Kumar, V., Sood, S.P., Jeberson, S. 2011. Relative efficiency of the maize and Imperata cylindrica mediated chromosome elimination approaches for induction of haploids of wheat-rye derivatives. Plant Breeding 130:192-194.

Mukai, Y., Okamoto, G., Kiryu, S., Takemoto, S., Sharma, S.K., Suzuki, G., Yamamoto, M. 2015. The D-genome plays a critical role in the formation of haploid Aegilops tauschii through Imperata cylindricamediated uniparental chromosome elimination. Nucleus 58:199-206.

Murashige, T., Skoog, S. 1962. A revised medium for rapid growth and bioassays with tobacco tissue cultures. Physiol. Plants 15:473-497.

Pratap, A., Chaudhary, H.K. 2012. Comparative effects of auxin analogues on induction of polyhaploids in triticale and triticale $\times$ wheat hybrids through wheat $\times$ maize system. The Indian J. Agric. Sci. 82:66-70.

Pratap, A., Sethi, G.S., Chaudhary, H.K. 2005. Relative efficiency of different Gramineae genera for haploid induction in triticale and triticale $\times$ wheat hybrids through chromosome elimination technique. Plant Breeding 124:147-153.

Rabinovich, S.V. 1998. Importance of wheat-rye translocations for breeding modern cultivars of Triticum aestivum L. Euphytica 100:323-340.

Silva, J.A.G. da, Carvalho, F.I.F. de, Silva, S.A., Barbieri, R.L., Marchioro, V.S., Lorencetti, C., Benin, G. 2002. Effect of pollination temperature to obtain haploid wheat embryos in intergeneric crosses. Revista Brasileira de Agrociencia 8:97-102. 
Suenaga, K. 1994. Doubled haploid system using the intergeneric crosses between wheat (Triticum aestivum) and maize (Zea mays). Bulletin National Institute of Agrobiological Resource 9:83-139.

Wedzony, M., Marcinska, I., Ponitka, A., Slusarkiewicz-Jarzina, A., Womna, J. 1998. Production of doubled haploids in Triticale ( $\times$ Triticosecale Wittmack) by means of crosses with maize (Zea mays) using Picloram and Dicamba. Plant Breeding 117:211-215. 